\title{
A case report of rhabdomyolysis caused by the use of roxadustat in the treatment of caused by renal anemia
}

qin yang $^{1}$ and xin wang ${ }^{1}$

${ }^{1}$ Affiliated Zhongshan Hospital of Dalian University

June 1, 2020

\begin{abstract}
More than 1.2 million people died from chronic kidney damage (CKD) globally, in 2017. Anemia is a common complication of CKD. Roxadustat is an oral hypoxia-inducible factor prolyl hydroxylase inhibitor that stimulates erythropoiesis and regulates iron metabolism. Roxadustat is often used to treat anemia caused by renal diseases. Its adverse effects include high blood pressure, myocardial infarction, heart failure, high potassium, and dizziness; however, there are no reports about rhabdomyolysis associated with roxadustat. One of our patients developed rhabdomyolysis after he was administered roxadustat to treat anemia caused by chronic renal failure. Physicians should be alert about the occurrence of rhabdomyolysis when roxadustat is used.
\end{abstract}

A case report of rhabdomyolysis caused by the use of roxadustat in the treatment of caused by renal anemia

Qin Yang ${ }^{1}$, Xin Wang ${ }^{1 *}$

1 Department of Nephrology, Affiliated Zhongshan Hospital of Dalian University. No. 6 Jiefang Street, Dalian, China

*Correspondence should be addressed to Xin Wang :

dl_wangxin1112@163.com

Hosted file

case report.docx available at https://authorea.com/users/328359/articles/455685-a-casereport-of-rhabdomyolysis-caused-by-the-use-of-roxadustat-in-the-treatment-of-caused-byrenal-anemia 

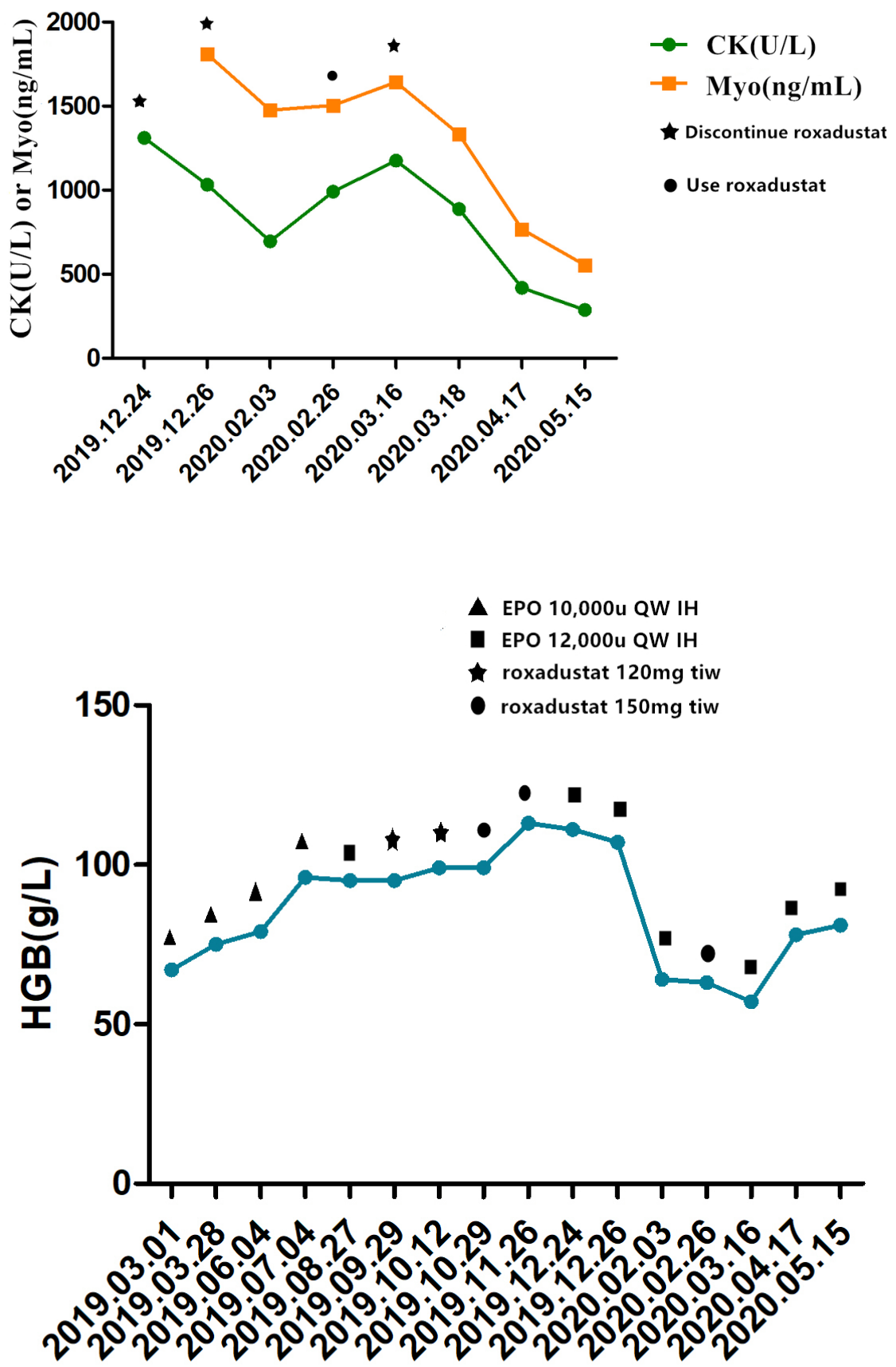